\title{
It's never too late to save a photoreceptor
}

\author{
James B. Hurley ${ }^{1,2}$ and Jennifer R. Chao' \\ 'Department of Ophthalmology and 2Department of Biochemistry, University of Washington, Seattle, Washington, USA.
}

\begin{abstract}
Recent gene therapy progress has raised the possibility that vision loss caused by inherited retinal degeneration can be slowed or prevented. Unfortunately, patients are not usually diagnosed until enough degeneration has occurred that the deterioration in vision is noticeable. Therefore, effective gene therapy must halt degeneration to stabilize and preserve any remaining vision. Gene therapy methods currently in human clinical trials rely on subretinal or intravitreal injections of adeno-associated virus to deliver the therapeutic gene. To date, long-term results in patients treated with subretinal injections for Leber congenital amaurosis have been mixed. Proposed limitations include variability in the gene delivery method and a possible point of no return, at which treatment would be ineffective. In this issue of the $J \mathrm{Cl}$, Koch et al. describe a well-controlled and precise mouse model for testing the ability of gene therapy to halt the progress of degeneration. Instead of viral-mediated therapeutic gene delivery, the authors induced expression of an integrated transgene at specific times during the course of photoreceptor degeneration. In Pde6bdeficient retina, this strategy halted degeneration, even when more than $70 \%$ of photoreceptors had already degenerated. The results of this study demonstrate that retinal degeneration can be stopped, even at late stages of disease.
\end{abstract}

\section{Congenital blinding diseases}

Hereditary blindness has been linked to at least 238 mutations that cause photoreceptor neurons in the retina to degenerate (1). For example, mutations in the gene that encodes the $\beta$ subunit of PDE6 cause a type of rod photoreceptor degeneration known as retinitis pigmentosa (RP) (2-4). PDE6 hydrolyzes cyclic GMP (cGMP) to 5'GMP in rods, is activated by light, and has a central role in phototransduction $(5,6)$. Defects in the enzymes that make cGMP from GTP also result in rod degeneration (7). For example, guanylyl cyclase deficiencies cause the inherited degenerative disease Leber congenital amaurosis type 1 (LCA1); however, the biochemical pathway that links abnormal cGMP metabolism is not fully understood (8).

\section{Why and when do mutant photoreceptors die?}

In some animal models of inherited retinal degeneration, the timing of individual rod death and the spatial distribution of dying rods appear to be random. In general, the rate at which rods degenerate in these models is simply proportional to the number of remaining rods, reminiscent of the spontaneous decay of radioactive isotopes. On the basis of these observations, Clark et al. (9) argued that degeneration of a photoreceptor is not caused by gradual accumulation of damage and instead proposed that degeneration is linked to a single random event that occurs in the context of a perturbed metabolic state on the threshold of insufficiency. Moreover, Clark and colleagues suggested that if those single events could be prevented (or if the

\section{Related Article: p. 3704}

threshold could be raised), then "the likelihood that a mutant neuron can be rescued by treatment is not diminished by age, although fewer cells will be available to rescue. Therefore, treatment at any stage of the illness is likely to confer benefit" (9).

This prediction was made in the year 2000, and since then, tremendous progress has been made in many aspects of gene therapy $(10,11)$. For example, one promising study (12) showed that injection of adeno-associated viruses (AAV) carrying a guanylyl cyclase-encoding gene halted degeneration of guanylyl cyclasedeficient rods in mice. This result suggested that AAV-mediated gene therapy could be used to treat human patients with guanylyl cyclase deficiencies. In fact, such studies are underway (13), but results have not been reported yet.

\section{What limits success of gene therapy in human retina?}

In human patients with retinal degeneration, gene therapy has so far largely focused on treatment of the inherited retinal degeneration LCA2, which is caused by an RPE65 deficiency. Clinical trials began more than seven years ago, and patient outcomes have been analyzed extensively. Retinal sensitivity in the treated regions of the retinae improved significantly (14-16); however, over time, retinae exhibited continued thinning, and it is apparent that degeneration was not effectively stopped (17-19).

The most pessimistic explanation of the lack of efficacy is that continued thinning of the retina reflects an inherent feature of gene therapy that limits the ability to halt the progress of degeneration. While this interpretation seems unlikely, it is a challenge to rigorously exclude the hypothesis that damage accumulates in photoreceptors to a point of no return, where the trajectory to death is irreversible (18). Alternatively, the continued retinal thinning may instead reflect only a technical limitation, such as insufficient delivery of the virus, or damage caused by temporary detachment of the neurosensory retina, especially in the subfoveal region, that pre- 

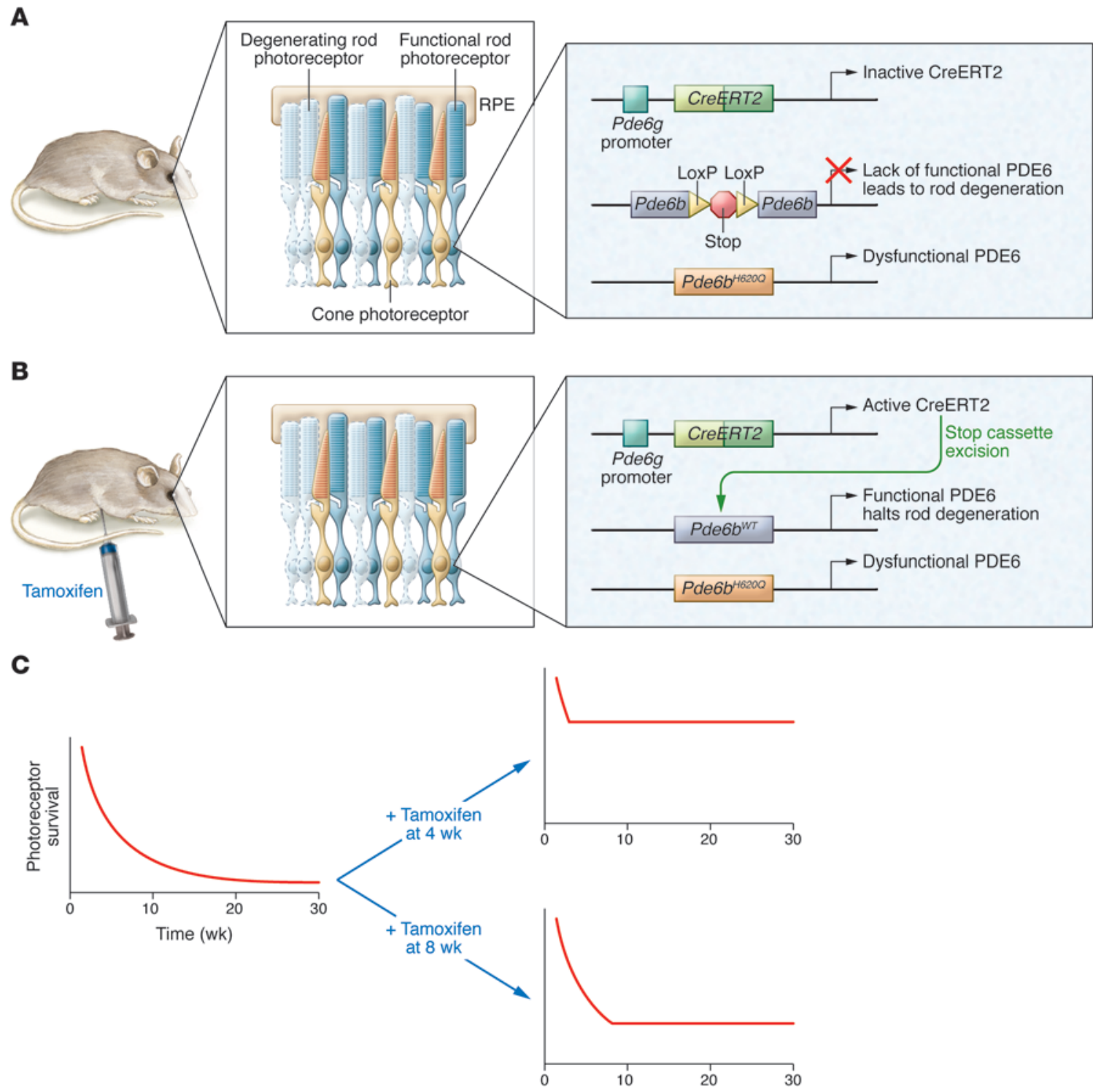

Figure 1. Strategy that allows evaluation of the point of no return for gene therapy to treat retinal degeneration. (A) Schematic of the genetic strategy developed by Koch et al. (21) to stimulate photoreceptor degeneration and evaluate the effect of rescuing the defect on demand by injecting the mouse with tamoxifen at different points during disease progression. The mouse has two different Pde6b alleles. One allele produces a mostly inactive protein as the result of an $\mathrm{H620Q}$ substitution. The other allele is normal, but it is interrupted by a floxed stop cassette. The very low PDE6 activity in the retinae of these mice causes rod photoreceptors to degenerate. (B) The Pde6g promoter is used to express CreERT2 in rods, and when the mouse is injected with tamoxifen, CreERT2 is activated and able to excise the stop cassette, resulting in expression of active PDE6. (C) In the absence of intervention, photoreceptors degenerate over time, resulting in vision loss. Tamoxifen-induced expression of active PDE6 at four or eight weeks of age halts degeneration. Although not shown in this schematic, the retinae continued to be stable out to 52 weeks of age.

vents an optimal response. Subretinal injections are inherently invasive and variable, and injected viruses do not reach the entire retina. Moreover, the reduction of trophic support that results from widespread cell loss in untreated areas could contribute to continued photoreceptor degeneration in treated areas. Some progress was made in animal models to address these issues by using AAV to rescue LCA2 mouse retinae at late stages of degeneration (20), but even those studies were hampered by the variability of subretinal injections. While gene therapy for LCA2 still looks promising at this point, the reasons for the partial rather than complete success are unclear.

\section{New strategy evaluates the point-of-no-return hypothesis}

In this issue, Koch et al. (21) describe a new, highly effective strategy to directly evaluate how well expression of a gene can halt degeneration. Importantly, this strategy was able to show that expression of Pde6b completely halts progression of photoreceptor degeneration in Pde6bdeficient retinae, regardless of the severity of photoreceptor cell loss. Specifically, Koch et al. devised an elegant genetic strategy that bypasses the need for AAV-mediated gene delivery (Figure 1). The authors engineered a mouse in which a therapeutic gene can be activated on demand in a 
degenerating retina. First, mice were generated with two different alleles of Pde6b, which encodes the PDE6 $\beta$ subunit. One allele carried a mutation that partially inactivates the enzyme and causes rods to degenerate. The other Pde6b allele was normal, except that it was interrupted by a floxed insertion that blocks Pde6b expression. This floxed Pde6b allele is designed to be inactive in the absence of Cre-driven recombination. These mice also harbor a rod-specific, tamoxifen-inducible Cre; therefore, expression of a normal PDE6 $\beta$ subunit within all remaining rod cells can be activated at any time by tamoxifen injection. Once Koch et al. confirmed that $P d e 6 b$ and Cre were expressed in rods as predicted, they used the engineered mice to test whether progression of degeneration could be halted by inducing normal $P d e 6 b$ expression at several different time points during the course of degeneration. Remarkably, expression of Pde6b completely impeded the progression of degeneration, even when less than $30 \%$ of photoreceptors remained.

Together, these findings show that restoration of a key protein can halt progression of a degenerative disease. This demonstration has significant implications for the treatment of retinal degeneration in humans, because patients generally do not seek care until after substantial photoreceptor loss has caused a noticeable deterioration of vision. These results are also consistent with the idea that widespread restoration of gene expression and rod photoreceptor rescue throughout the retina prevents further cone and rod degeneration that otherwise would occur by non-cell-autonomous mechanisms.

\section{Etiology matters}

The ability of induced gene expression to halt degeneration is important; however, this strategy may or may not apply to all types of retinal degeneration. For example, a key difference between degeneration in $\mathrm{RP}, \mathrm{LCA} 1$, and LCA2 is that the defect originates in photoreceptors in RP and LCA1, whereas it originates in the retinal pigment epithelium (RPE) in LCA2. Additionally, RP- and LCA1-associated mutations perturb guanine nucleotide metabolism, whereas LCA2-causing mutations disrupt the visual cycle and cause massive accumulation of retinyl esters in the RPE (22).

The diverse etiologies of these and other different types of degeneration could influence how well different inherited retinal diseases respond to gene therapy. The strategy that Koch et al. devised (21) is well controlled and precise and bypasses the uncertainty and variability associated with AAV injections. Gene therapists should have a tool that helps them predict how well each type of degenerative disease will respond to gene therapy and perhaps how to optimize the response. When inducible Cre becomes available in other cell types such as RPE, the genetic strategy devised by Koch et al. could provide an effective way to make those types of predictions for LCA2 and other types of retinal degeneration.

\section{Acknowledgments}

The authors acknowledge support from $\mathrm{NIH}$ grants EY06641 (to J.B. Hurley), EY017863 (to J.B. Hurley), and EY019714 (to J.R. Chao). We thank John Flannery for helpful discussions.

Address correspondence to: James B. Hurley, Department of Biochemistry, UW Box 357350, University of Washington, 1705 NE Pacific St., Seattle, Washington 98195, USA. Phone: 206.543.2871; E-mail:jbhhh@uw.edu.

1. Daiger SP. RetNET: Summaries of Genes and Loci Causing Retinal Diseases. University of Texas Health Science Center Web site. https://sph.uth.edu/RetNet/sum-dis.htm. Updated June 19, 2015. Accessed July 27, 2015, 2015

2. Bowes C, Li T, Danciger M, Baxter LC, Applebury ML, Farber DB. Retinal degeneration in the rd mouse is caused by a defect in the beta subunit of rod cGMP-phosphodiesterase. Nature. 1990;347(6294):677-680.

3. McLaughlin ME, Ehrhart TL, Berson EL, Dryja TP. Mutation spectrum of the gene encoding the beta subunit of rod phosphodiesterase among patients with autosomal recessive retinitis pigmentosa. Proc Natl Acad Sci U S A. 1995;92(8):3249-3253.

4. Pittler SJ, Baehr W. Identification of a nonsense mutation in the rod photoreceptor cGMP phosphodiesterase beta-subunit gene of the rd mouse. Proc Natl Acad Sci U S A. 1991;88(19):8322-8326.

5. Hurley JB. Molecular properties of the cGMP cascade of vertebrate photoreceptors. Annu Rev Physiol. 1987;49:793-812.

6. Zhang X, Cote RH. cGMP signaling in vertebrate retinal photoreceptor cells. Front Biosci. 2005;10:1191-1204.

7. Boye SE. Insights gained from gene therapy in animal models of retGC1 deficiency. Front $\mathrm{Mol}$ Neurosci. 2014;7:43.

8. Sancho-Pelluz J, et al. Photoreceptor cell death mechanisms in inherited retinal degeneration. Mol Neurobiol. 2008;38(3):253-269.

9. Clarke G, et al. A one-hit model of cell death in inherited neuronal degenerations. Nature. 2000;406(6792):195-199.

10. Boye SE, Boye SL, Lewin AS, Hauswirth WW. A comprehensive review of retinal gene therapy. Mol Ther. 2013;21(3):509-519.

11. Thompson DA, et al. Advancing therapeutic strategies for inherited retinal degeneration: recommendations from the Monaciano Symposium. Invest Ophthalmol Vis Sci. 2015;56(2):918-931.

12. Boye SL, et al. AAV-mediated gene therapy in the guanylate cyclase (RetGC1/RetGC2) double knockout mouse model of Leber congenital amaurosis. Hum Gene Ther. 2013;24(2):189-202.

13. Boye SE. Leber congenital amaurosis caused by mutations in GUCY2D. Cold Spring Harb Perspect Med. 2015;5(1):a017350.

14. Bainbridge JW, et al. Effect of gene therapy on visual function in Leber's congenital amaurosis. N Engl J Med. 2008;358(21):2231-2239.

15. Hauswirth WW, et al. Treatment of leber congenital amaurosis due to RPE65 mutations by ocular subretinal injection of adeno-associated virus gene vector: short-term results of a phase I trial. Hum Gene Ther. 2008;19(10):979-990.

16. Maguire AM, et al. Safety and efficacy of gene transfer for Leber's congenital amaurosis. $N$ Engl JMed. 2008;358(21):2240-2248.

17. Bainbridge JW, et al. Long-term effect of gene therapy on Leber's congenital amaurosis. $N$ Engl JMed. 2015;372(20):1887-1897.

18. Cepko CL, Vandenberghe LH. Retinal gene therapy coming of age. Hum Gene Ther. 2013;24(3):242-244.

19. Jacobson SG, et al. Improvement and decline in vision with gene therapy in childhood blindness. N Engl J Med. 2015;372(20):1920-1926.

20. Li X, et al. Gene therapy rescues cone structure and function in the 3-month-old rd12 mouse: a model for midcourse RPE65 leber congenital amaurosis. Invest Ophthalmol Vis Sci. 2011;52(1):7-15.

21. Koch SF, et al. Halting progressive neurodegeneration in advanced retinitis pigmentosa. J Clin Invest. 2015;125(9):3704-3713.

22. Redmond TM, et al. Rpe 65 is necessary for production of 11-cis-vitamin A in the retinal visual cycle. Nat Genet. 1998;20(4):344-351. 